\title{
Synthesis and structure-activity relationship of 8-substituted protoberberine derivatives as a novel class of antitubercular agents
}

\author{
Ying-Hong Li ${ }^{1 \dagger}$, Hai-Gen Fu' ${ }^{1 \dagger}$, Feng Su${ }^{2}$, Li-Mei Gao ${ }^{1}$, Sheng Tang ${ }^{1}$, Chong-Wen Bi ${ }^{1}$, Yu-Huan Li',
} Yan-Xiang Wang ${ }^{1 *}$ and Dan-Qing Song ${ }^{1 *}$

\begin{abstract}
Background: The emergence of multi-drug resistant tuberculosis (MDR-TB) has heightened the need for new chemical classes and innovative strategies to tackle TB infections. It is urgent to discover new classes of molecules without cross-resistance with currently used antimycobacterial drugs.

Results: Eighteen new 8-substituted protoberberine derivatives were synthesized and evaluated for their anti-mycobacterial activities against Mycobacterium tuberculosis (M. tuberculosis) strain $\mathrm{H}_{37} \mathrm{Rv}$. Among them, compound $7 \mathrm{~g}$ was the most effective antitubercular agent with minimum inhibitory concentration (MIC) of $0.5 \mu \mathrm{g} / \mathrm{mL}$. Moreover, it also afforded a potent antitubercular effect against clinically isolated MDR strains of M. tuberculosis with MICs ranging from 0.25 to $1.0 \mu \mathrm{g} / \mathrm{mL}$, suggesting a novel mode of action.

Conclusions: The structure-activity relationship (SAR) analysis revealed that introduction of a substituent at the 8-position in pseudoprotoberberine, especially an $n$-decyl, could significantly enhance the anti-TB activity. We consider 8-n-decylberberines to be a novel family of anti-tubercular agents with an advantage of inhibiting MDR strains of $M$. tuberculosis.
\end{abstract}

Keywords: 8-Substituted-protoberberine, Antitubercular, Structure-activity relationship, Drug-resistance

\section{Background}

Currently, one third of the world's population is infected with Mycobacterium tuberculosis (M. tuberculosis) [1]. It is anticipated that there will be about 8.9-9.9 million new and relapse tuberculosis (TB) cases this year, more than in any other year in history [2,3]. The limited effectiveness and long-term treatment lead to poor patient compliance, which often causes multi-drug resistant (MDR) and extensively-drug-resistant (XDR). The emergence of new cases, the increased incidence of MDR strains of $M$. tuberculosis, the adverse effects of first-line anti-TB drugs isoniazid (INH) and rifampin (RIF) [4,5], and the increased incidence of TB associated with HIV infections [6-8] have led to renewed research interest in discovering novel anti-TB drugs. Especially, the

\footnotetext{
* Correspondence: lionking_0421@hotmail.com; songdanqingsdq@hotmail.com ${ }^{\dagger}$ Equal contributors

'Institute of Medicinal Biotechnology, Chinese Academy of Medical Sciences and Peking Union Medical College, Beijing 100050, China

Full list of author information is available at the end of the article
}

emergence of MDR-TB and of the virtually untreatable MDR-TB has heightened the need for new chemical class and innovative strategies to tackle TB infections. However, truly novel antitubercular drugs other than repurposed drugs have not been developed since the 1970s $[9,10]$. Though new anti-TB drug Bedaquiline was just approved by FDA last year [11], there is still an urgent need to discover new classes of molecules without crossresistance with currently used antimycobacterial drugs.

We have identified 13-substituted protoberberine derivatives to be a novel family of anti-TB agents [12] with poor solubility. The primary structure-activity relationship (SAR) indicated that the berberine ring (BBR, 1, Figure 1) might be beneficial for keeping good antitubercular activity. In our ongoing efforts to discovering new anti-TB agents, we turned our SAR analysis on the substituents at the 8-position of BBR derivatives, by which nitrogen ion at the 7-position might be blocked by the 8-substituents with bigger volume, thereby enhancing the solubility of
() Chemistry Central

(C) 2013 Li et al.; licensee Chemistry Central Ltd. This is an Open Access article distributed under the terms of the Creative Commons Attribution License (http://creativecommons.org/licenses/by/2.0), which permits unrestricted use, distribution, and reproduction in any medium, provided the original work is properly cited. 


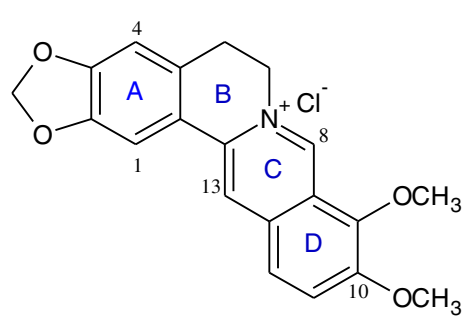

BBR (1)<smiles>COc1cc2cc3[n+](cc2cc1OC)-c1cc2c(cc1CC[Cl+]2)OCO3</smiles>

Pesudoberberine (2)<smiles>COc1cc2c(cc1OC)-c1cc3ccc(OC)c(OC)c3c[n+]1CC2</smiles>

Palmatine (3)

Figure 1 Chemical structures of compounds 1, 2 and 3.

this kind of compounds. Based on this strategy, several 8 -substituted protoberberine derivatives $(\mathbf{6 a}-\mathbf{e})$ were designed, semi-synthesized and evaluated for their antimycobacterial activity against $M$. tuberculosis strain $\mathrm{H}_{37} \mathrm{Rv}$. Furthermore, by replacing $\mathbf{1}$ with pseudoberberine (2, Figure 1 ) or palmatine (3, Figure 1 ) core, two natural products extracted from Chinese herb Huanglian, by which a group of new 8-substituted pseudoberberine $(7 \mathbf{a}-\mathbf{h})$ or palmatine $(\mathbf{8} \mathbf{a}-\mathbf{e})$ derivatives was generated for testing. Herein, eighteen 8-substituted protoberberine derivatives (Figure 2) were designed and synthesized, and their anti-mycobacterial effects were evaluated afterwards.

\section{Results and discussion}

\section{Chemistry}

Eighteen target compounds were synthesized with commercially available 1, 3 or $\mathbf{2}$ (synthesized in our laboratory) $[13,14]$ as the starting material as described in Scheme 1. The Grignard reagents were first prepared with Mg turnings and the corresponding alkyl and aryl iodide in absolute ether under $\mathrm{N}_{2}$ protection. The key intermediates dihydroberberine, dihydropseudoberberine or dihydropalmatine (4) were obtained via nucleophilic substitution of newly synthesized Grignard reagents with 1, 2 or 3 under $\mathrm{N}_{2}$ protection, respectively $[15,16]$. Then, the intermediate 4 was oxidized using bromine as a oxidizing agent in HOAc at refluxing temperature to yield the 8-substituted berberine bromate 5 , which was converted into the corresponding chloride 6-8 with $\mathrm{AgCl}$ in $\mathrm{MeOH}$ at room temperature. Finally, the desired products in series 6, 7 and $\mathbf{8}$ were purified by flash column chromatography using methanol/dichloromethane as the gradient eluent with overall yields of $72 \%-81 \%$.

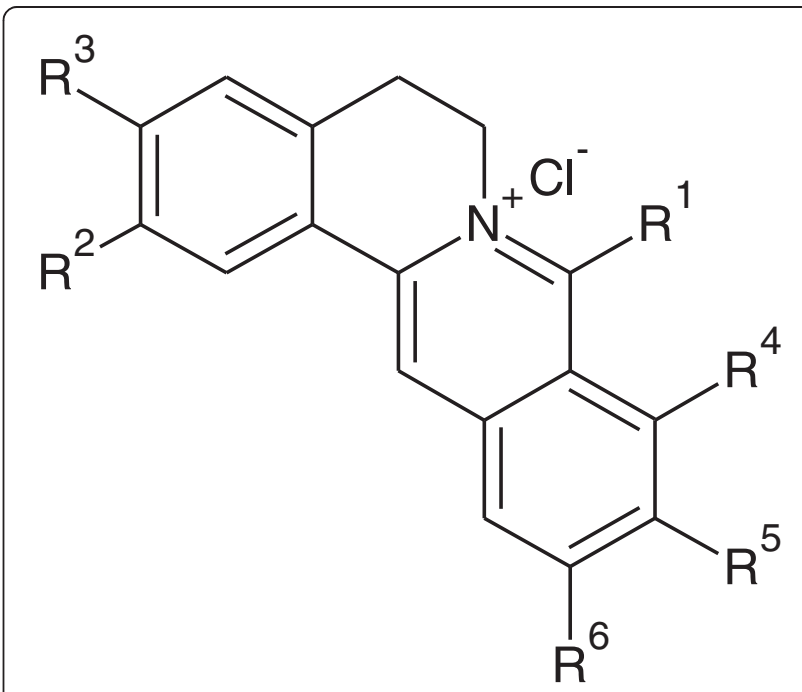

Figure 2 General structural formula of 8-substituted BBR derivatives. 


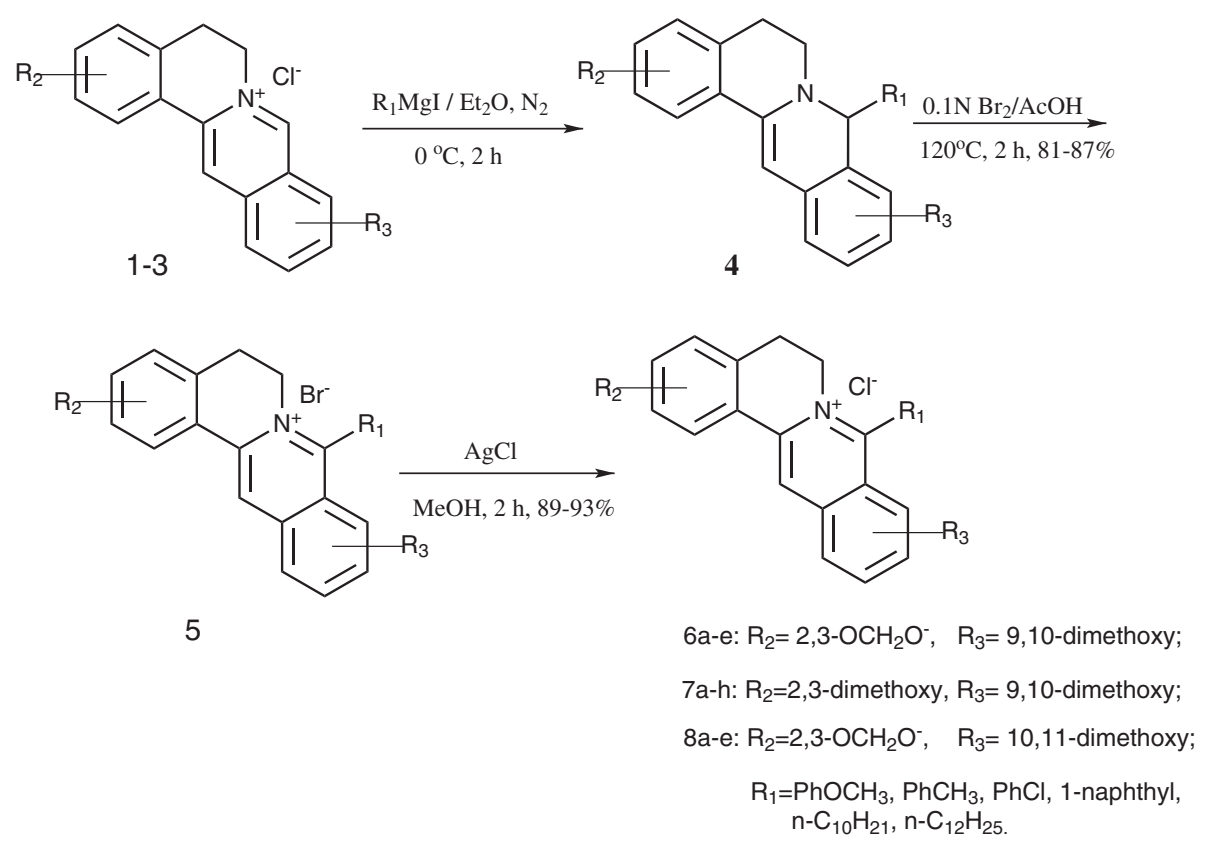

Scheme 1 The synthetic route of the target compounds.

\section{Biological activity and SAR analysis of 8-substituted} protoberberine derivatives for anti-mycobacterial activity Our SAR strategy was first focused on the modifications of the substituents at the 8-position in BBR. According to our previous SAR results, several lipophilic groups including $p$-methoxyphenyl (6a), m-methoxyphenyl (6b), $p$-methylphenyl (6c), 1-naphthyl (6d) and $n$-decyl (6e) were introduced into the 8-position aiming to improve the cLogP value (Table 1), thereby enhancing the antimycobacterial activity. Among these analogues, compound 6e possessing an $n$-decyl afforded the hightest antibacterial activity with a MIC of $2.0 \mu \mathrm{g} / \mathrm{mL}$ against M. tuberculosis. The results supported that the increased cLogP value might be helpful for enhancing the anti-TB activity of this kind of compounds.

In order to further explore the influence of the BBR core, a variety of lipophilic substituents were attached to the 8 -position of 2 , by which the 8 new 8 -substituted pseudoberberine classes $(7 \mathbf{a}-\mathbf{h})$ were generated for testing. The results showed (Table 1 ) that the majority of them $(\mathbf{7 b}-\mathbf{h})$ exhibited potential anti-mycobacterial activities with MICs ranging from $0.5 \mu \mathrm{g} / \mathrm{mL}$ to $2.0 \mu \mathrm{g} / \mathrm{mL}$. It seems that the 10,11-dimethoxy on the ring $\mathrm{D}$ would be beneficial for their binding affinity to the target molecular. Compound $\mathbf{7 g}$ bearing an $n$-decyl afforded the best anti-TB activity with a MIC of $0.5 \mu \mathrm{g} / \mathrm{mL}$. Similarly, the lipophilic side-chains were also introduced to the same position in $\mathbf{3}$, and then 5 new 8-substituted palmatine analogues $(\mathbf{8 a}-\mathbf{e})$ were made. As expected, compound $8 \mathbf{e}$ with an $n$-decyl at position 8 had the most potent activity with an MIC of $1.0 \mu \mathrm{g} / \mathrm{mL}$. It was deduced that an $n$-decyl at the 8 -position would improve the anti-mycobacterial activity as regarding to this kind of compounds. The pesudoberberine ring might be beneficial for the antimycobacterial activity, and thus the representative compounds in 7 series were chosen for further investigation.

\section{Anti-resistance TB effect of $7 g$ and $7 f$}

As compound $7 \mathbf{g}$ bearing an $n$-alkyl at position 8 possessed an excellent activity against drug-susceptible $M$. tuberculosis strain $\mathrm{H}_{37} \mathrm{Rv}$, it was selected to test the anti-TB activity against MDR strains. In this experiment, M. tuberculosis strains 87, 192, 262 and 266 isolated from the patient infected with tuberculosis in China, were resistant to both RIF and INH. RIF and INH showed a decreased activity against the drugresistant stains partially or completely with MIC ranges between 2 and $>32 \mu \mathrm{g} / \mathrm{mL}$ (Table 2), while compound $7 \mathbf{g}$ afforded a potential effect against MDR strains with comparable MIC ranges of $0.25-1 \mu \mathrm{g} / \mathrm{mL}$. In addition, compound 7f possessing an aromatic moiety at the 8position in 7 series afforded a moderate $\operatorname{cLog} \mathrm{P}$ value $(\mathrm{cLog} \mathrm{P}=1.60)$ and then was chosen to evaluate for the drug-resistant strains as well. As described in Table 2, 7f showed an equivalent potency against the drugsusceptible strain $\mathrm{H}_{37} \mathrm{Rv}$ and multidrug-resistance isolates of $M$. tuberculosis strains 257, 373, 559 and 164 with a MIC range between 2 and $4 \mu \mathrm{g} / \mathrm{mL}$ as well. The results indicated that $7 \mathbf{g}$ and $7 \mathbf{f}$ were effective for drug- 
Table 1 SAR of 8-substituted protoberberine analogous against $M$. tuberculosis strain $\mathrm{H}_{37} \mathrm{Rv}$

\begin{tabular}{|c|c|c|c|c|c|c|c|c|}
\hline Compd $^{\mathrm{a}}$ & $\mathrm{R}^{1}$ & $R^{2}$ & $R^{3}$ & $R^{4}$ & $R^{5}$ & $R^{6}$ & $\mathrm{MIC}^{\mathrm{b}}$ & $\operatorname{cLog} \mathrm{P}^{\mathrm{c}}$ \\
\hline 1 & & & & & & & $>128$ & -0.77 \\
\hline 2 & & & & & & & 128 & -1.08 \\
\hline 3 & & & & & & & $>128$ & -0.77 \\
\hline $6 a$ & $\mathrm{PhOCH}_{3}-p$ & & $\mathrm{OCH}_{2} \mathrm{O}$ & $\mathrm{OCH}_{3}$ & $\mathrm{OCH}_{3}$ & $\mathrm{H}$ & 32 & 0.35 \\
\hline $6 b$ & $\mathrm{PhOCH}_{3}-\mathrm{m}$ & & $\mathrm{OCH}_{2} \mathrm{O}$ & $\mathrm{OCH}_{3}$ & $\mathrm{OCH}_{3}$ & $\mathrm{H}$ & 16 & 0.35 \\
\hline $6 c$ & $\mathrm{PhCH}_{3}-p$ & & $\mathrm{OCH}_{2} \mathrm{O}$ & $\mathrm{OCH}_{3}$ & $\mathrm{OCH}_{3}$ & $\mathrm{H}$ & 16 & 0.93 \\
\hline $6 d$ & 1-naphthyl & & $\mathrm{OCH}_{2} \mathrm{O}$ & $\mathrm{OCH}_{3}$ & $\mathrm{OCH}_{3}$ & $\mathrm{H}$ & 8 & 1.60 \\
\hline $6 e$ & $n-\mathrm{C}_{10} \mathrm{H}_{21}$ & & $\mathrm{OCH}_{2} \mathrm{O}$ & $\mathrm{OCH}_{3}$ & $\mathrm{OCH}_{3}$ & $\mathrm{H}$ & 2 & 3.29 \\
\hline $7 a$ & $\mathrm{PhOCH}_{3}-p$ & & $\mathrm{OCH}_{2} \mathrm{O}$ & $\mathrm{H}$ & $\mathrm{OCH}_{3}$ & $\mathrm{OCH}_{3}$ & 4 & 0.35 \\
\hline $7 b$ & $\mathrm{PhOCH}_{3}-m$ & & $\mathrm{OCH}_{2} \mathrm{O}$ & $\mathrm{H}$ & $\mathrm{OCH}_{3}$ & $\mathrm{OCH}_{3}$ & 2 & 0.35 \\
\hline $7 c$ & $\mathrm{PhCH}_{3}-p$ & & $\mathrm{OCH}_{2} \mathrm{O}$ & $\mathrm{H}$ & $\mathrm{OCH}_{3}$ & $\mathrm{OCH}_{3}$ & 2 & 0.93 \\
\hline $7 d$ & $\mathrm{PhCl}-p$ & & $\mathrm{OCH}_{2} \mathrm{O}$ & $\mathrm{H}$ & $\mathrm{OCH}_{3}$ & $\mathrm{OCH}_{3}$ & 1 & 1.14 \\
\hline $7 e$ & $\mathrm{PhCl}-m$ & & $\mathrm{OCH}_{2} \mathrm{O}$ & $\mathrm{H}$ & $\mathrm{OCH}_{3}$ & $\mathrm{OCH}_{3}$ & 2 & 1.14 \\
\hline $7 f$ & 1-naphthyl & & $\mathrm{OCH}_{2} \mathrm{O}$ & $\mathrm{H}$ & $\mathrm{OCH}_{3}$ & $\mathrm{OCH}_{3}$ & 2 & 1.60 \\
\hline $7 g$ & $n-\mathrm{C}_{10} \mathrm{H}_{21}$ & & $\mathrm{OCH}_{2} \mathrm{O}$ & $\mathrm{H}$ & $\mathrm{OCH}_{3}$ & $\mathrm{OCH}_{3}$ & 0.5 & 3.29 \\
\hline $7 \mathrm{~h}$ & $n-\mathrm{C}_{12} \mathrm{H}_{25}$ & & $\mathrm{OCH}_{2} \mathrm{O}$ & $\mathrm{H}$ & $\mathrm{OCH}_{3}$ & $\mathrm{OCH}_{3}$ & 1 & 4.35 \\
\hline $8 a$ & $\mathrm{PhOCH}_{3}-p$ & $\mathrm{OCH}_{3}$ & $\mathrm{OCH}_{3}$ & $\mathrm{OCH}_{3}$ & $\mathrm{OCH}_{3}$ & $\mathrm{H}$ & 16 & 0.04 \\
\hline $8 b$ & $\mathrm{PhOCH}_{3}-\mathrm{m}$ & $\mathrm{OCH}_{3}$ & $\mathrm{OCH}_{3}$ & $\mathrm{OCH}_{3}$ & $\mathrm{OCH}_{3}$ & $\mathrm{H}$ & 16 & 0.04 \\
\hline $8 c$ & $\mathrm{PhCH}_{3}-p$ & $\mathrm{OCH}_{3}$ & $\mathrm{OCH}_{3}$ & $\mathrm{OCH}_{3}$ & $\mathrm{OCH}_{3}$ & $\mathrm{H}$ & 16 & 0.62 \\
\hline $8 d$ & 1-naphthyl & $\mathrm{OCH}_{3}$ & $\mathrm{OCH}_{3}$ & $\mathrm{OCH}_{3}$ & $\mathrm{OCH}_{3}$ & $\mathrm{H}$ & 4 & 1.29 \\
\hline $8 e$ & $n-\mathrm{C}_{10} \mathrm{H}_{21}$ & $\mathrm{OCH}_{3}$ & $\mathrm{OCH}_{3}$ & $\mathrm{OCH}_{3}$ & $\mathrm{OCH}_{3}$ & $\mathrm{H}$ & 1 & 2.98 \\
\hline RIF & & & & & & & 0.0625 & 3.90 \\
\hline $\mathrm{INH}$ & & & & & & & 0.0625 & -1.12 \\
\hline
\end{tabular}

${ }^{a}:$ General structural formular is shown in Figure 2.

b: MIC: minimum inhibitory concentration.

c: Chemoffice Ultra 11.0 (Cambridge office).

RIF: rifampin; INH: isoniazid.

susceptible $M$. tuberculosis as well as MDR strains isolated from TB patients in China, suggesting a mode of action different from currently used anti-TB drugs.

\section{Cytotoxicity of $7 \mathrm{~g}$ and $7 \mathrm{f}$ in Vero and MRC-5 cells}

Both of compounds $\mathbf{7 g}$ and $\mathbf{7 f}$ were further tested their cytotoxicity in African green monkey kidney (Vero) and human lung fibroblast (MRC-5) cells with MTT assay. Cytotoxicity activity was expressed with $\mathrm{CC}_{50}$ value, and

Table 2 In vitro antitubercular activities of compounds $7 f$ and $\mathbf{7 g}$ against MDR strains of $M$. tuberculosis ${ }^{a}$

(MIC: $\mu \mathrm{g} / \mathrm{mL}$ )

\begin{tabular}{llllll}
\hline Compd & $\mathbf{2 5 7 / 8 7}$ & $\mathbf{3 7 3 / 1 9 2}$ & $\mathbf{5 5 9 / 2 6 2}$ & $\mathbf{1 6 4 / 2 6 6}$ & $\mathbf{H}_{\mathbf{3}} \mathbf{R \mathbf { v }}$ \\
\hline $\mathbf{7 f}^{\mathbf{b}}$ & 2 & 4 & 2 & 2 & 2 \\
$\mathbf{7 g}^{\mathbf{c}}$ & 0.5 & 0.25 & 0.5 & 1 & 0.5 \\
$\mathrm{RIF}$ & $>32$ & $>32$ & $>32$ & $>32$ & 0.0625 \\
$\mathrm{INH}$ & 2 & 4 & 2 & 2 & 0.0625 \\
\hline
\end{tabular}

${ }^{a}$ : MDR strains were isolated from patients with tuberculosis in China.

b: MIC values against MDR strains 257, 373, 559 and 164 .

c: MIC values against MDR strains 87, 192, 262 and 266. the selectivity index (SI), as an important therapeutic indication, was calculated as the ratio of $\mathrm{CC}_{50}$ to MIC value. Anti-TB effect of compounds $\mathbf{7 g}$ and $\mathbf{7 f}$ was evaluated by combining their MIC with SI values. As described in Table 3, compound $\mathbf{7 g}$ showed a moderate SI value of 10.3 and 17.6 in Vero and MRC-5 cells, respectively.

\section{Experimental}

\section{Instruments}

Melting point (m.p.) was uncorrected and recorded on a Mettler Toledo MP90 melting point apparatus. ${ }^{1} \mathrm{H}$ NMR

Table 3 Cytotoxicity ${ }^{a}$ activities of compounds $7 \mathbf{f}$ and $\mathbf{7 g}$ in Vero and MRC-5 cells

\begin{tabular}{lllll}
\hline Compd & \multicolumn{2}{c}{ Vero } & \multicolumn{2}{c}{ MRC-5 } \\
\hline & $\mathrm{CC}_{50}(\mathrm{ug} / \mathrm{ml})$ & $\mathrm{Sl}^{\mathrm{b}}$ & $\mathrm{CC}_{50}(\mathrm{ug} / \mathrm{ml})$ & $\mathrm{SI}$ \\
$\mathbf{7 f}$ & $14.72 \pm 2.02$ & 7.37 & $10.18 \pm 2.78$ & 5.09 \\
$\mathbf{7 g}$ & $5.15 \pm 1.29$ & 10.3 & $8.78 \pm 0.48$ & 17.6 \\
\hline
\end{tabular}

${ }^{a}$ : Cytotoxic concentration required to inhibit Vero or MRC-5 cell growth by $50 \%$.

b: Selectivity index $(\mathrm{SI})$ value equaled to $\mathrm{CC}_{50} / \mathrm{MIC}$. 
and ${ }^{13} \mathrm{C}$ NMR spectra were recorded on Varian $400 \mathrm{MHz}$ spectrometer. Chemical shift was reported relative to internal tetramethylsilane $(\delta 0.00 \mathrm{ppm})$ or $\left(\mathrm{CD}_{3}\right)_{2} \mathrm{SO}(\delta 2.50$ $\mathrm{ppm})$ for ${ }^{1} \mathrm{H}$ and $\left(\mathrm{CD}_{3}\right)_{2} \mathrm{SO}(\delta 39.5 \mathrm{ppm})$ for ${ }^{13} \mathrm{C}$. ESI high-resolution mass spectra (HRMS) were recorded on an Autospec UItima-TOF mass spectrometer (Micromass UK Ltd, Manchester, UK). Flash chromatography was performed on CombiflashRf 200 (Teledyne, Nebraska, USA), particle size $0.038 \mathrm{~mm}$.

\section{General procedure to obtain final 8-substituted protoberberine derivatives}

Grignard reagents were prepared via Magnesium turnings (3.8 g) with the corresponding alkyl and aryl iodides $(0.13$ $\mathrm{mol})$ in absolute ether $(100 \mathrm{~mL})$ at $0^{\circ} \mathrm{C}$. The synthesized Grignard reagents were added to the suspension of dry $\mathbf{1}$, 2 or $3(0.03 \mathrm{~mol})$ in absolute ether $(100 \mathrm{~mL})$ dropwise under $\mathrm{N}_{2}$ protection at $0^{\circ} \mathrm{C}[15,16]$. After refluxing for $2 \mathrm{~h}$, saturated $\mathrm{NH}_{4} \mathrm{Cl}$ solution $(200 \mathrm{~mL}$ ) was added to quench the reaction. The aqueous phase was extracted with ethyl acetate $(3 \times 100 \mathrm{~mL})$ and the combined organic layers were washed with saturated brine $(100 \mathrm{~mL})$ and dried $\left(\mathrm{Na}_{2} \mathrm{SO}_{4}\right)$. The mixture was concentrated in vacuo to give 4. Then, bromine solution $(1 \mathrm{~N} / \mathrm{HOAc}, 30 \mathrm{~mL})$ was added to oxidize compound 4 in HOAc at refluxing temperature to generate the 8-substituted BBR derivatives bromate 5, which was treated with excessive $\mathrm{AgCl}$ in $\mathrm{MeOH}$ at room temperature thus converted into the corresponding chloride form. Finally, the desired products in series 6, 7 and 8 were purified by flash column chromatography using methanol/ dichloromethane as the gradient eluent with overall yields of $72 \%-81 \%$.

\section{8-(p-Methoxy)phenylprotoberberine choride (6a)}

Yield: 78\%; Brown solid; mp 220-221 ${ }^{\circ} \mathrm{C} ;{ }^{1} \mathrm{H}$ NMR: $\delta 9.04$ $(\mathrm{s}, 1 \mathrm{H}), 8.23(\mathrm{~d}, J=9.2 \mathrm{~Hz}, 1 \mathrm{H}), 8.11(\mathrm{~d}, J=9.2 \mathrm{~Hz}, 1 \mathrm{H})$, $7.84(\mathrm{~s}, 1 \mathrm{H}), 7.56(\mathrm{~d}, J=8.8 \mathrm{~Hz}, 2 \mathrm{H}), 7.22(\mathrm{~d}, J=8.8 \mathrm{~Hz}$, 2H), $7.04(\mathrm{~s}, 1 \mathrm{H}), 6.18(\mathrm{~s}, 2 \mathrm{H}), 4.26(\mathrm{t}, J=6.8 \mathrm{~Hz}, 2 \mathrm{H}), 4.00$ (s, 3H), $3.98(\mathrm{~s}, 3 \mathrm{H}), 3.20(\mathrm{~s}, 3 \mathrm{H}), 3.01(\mathrm{t}, J=6.8 \mathrm{~Hz}, 2 \mathrm{H})$; ${ }^{13}$ C NMR: $\delta$ 172.40, 160.65, 156.96, 153.08, 150.26, 148.18, $145.55,138.47,133.66,131.30,129.73,126.90,126.30$, $124.72,122.84,121.88,121.81,114.41,110.01,108.36$, $106.31,102.56,61.09,57.44,55.90,52.26,26.67$; HRMSESI: $m / z$ calcd $442.16490 \mathrm{C}_{27} \mathrm{H}_{24} \mathrm{NO}_{5} \mathrm{Cl}[\mathrm{M}-\mathrm{Cl}]^{+}$, found 442.16476 .

\section{8-(m-Methoxy)phenylprotoberberine choride ( $6 b)$}

Yield: 79\%; Orange solid; mp $206-207^{\circ} \mathrm{C} ;{ }^{1} \mathrm{H}$ NMR: $\delta$ $9.07(\mathrm{~s}, 1 \mathrm{H}), 8.24(\mathrm{~d}, J=9.2 \mathrm{~Hz}, 1 \mathrm{H}), 8.11(\mathrm{~d}, J=9.2 \mathrm{~Hz}$, $1 \mathrm{H}), 7.85(\mathrm{~s}, 1 \mathrm{H}), 7.58(\mathrm{t}, J=8.0 \mathrm{~Hz}, 1 \mathrm{H}), 7.20-7.26(\mathrm{~m}$, $3 \mathrm{H}), 7.04(\mathrm{~s}, 1 \mathrm{H}), 6.18(\mathrm{~s}, 2 \mathrm{H}), 4.27(\mathrm{t}, J=6.4 \mathrm{~Hz}, 2 \mathrm{H})$, $4.00(\mathrm{~s}, 3 \mathrm{H}), 3.81(\mathrm{~s}, 3 \mathrm{H}), 3.26(\mathrm{~s}, 3 \mathrm{H}), 3.02(\mathrm{t}, J=6.4 \mathrm{~Hz}$, $2 \mathrm{H}) ;{ }^{13} \mathrm{C}$ NMR: $\delta 172.49,159.61,156.25,153.02,150.31$, $148.20,145.40,138.37,136.15,133.72,131.28,130.41$,
$126.41,124.65,122.44,121.76,120.09,115.85,113.70$, 108.39, 106.29, 102.58, 61.15, 57.45, 55.87, 52.35, 26.66; HRMS-ESI: $m / z$ calcd $442.16490 \mathrm{C}_{27} \mathrm{H}_{24} \mathrm{NO}_{5} \mathrm{Cl}[\mathrm{M}-\mathrm{Cl}]^{+}$, found 442.16466 .

\section{8-(p-Methyl)phenylprotoberberine choride (6c)}

Yield: 76\%; Orange solid; mp $158-160^{\circ} \mathrm{C}$ (decomp); ${ }^{1} \mathrm{H}$ NMR: $\delta 9.05$ (s, 1H), 8.23 (d, $J=9.2 \mathrm{~Hz}, 1 \mathrm{H}), 8.12$ (d, $J=$ $9.2 \mathrm{~Hz}, 1 \mathrm{H}), 7.85(\mathrm{~s}, 1 \mathrm{H}), 7.52(\mathrm{~d}, J=8.4 \mathrm{~Hz}, 2 \mathrm{H}), 7.47$ $(\mathrm{d}, J=8.4 \mathrm{~Hz}, 2 \mathrm{H}), 7.03(\mathrm{~s}, 1 \mathrm{H}), 6.18(\mathrm{~s}, 2 \mathrm{H}), 4.23(\mathrm{t}, J=$ $6.4 \mathrm{~Hz}, 2 \mathrm{H}), 3.99(\mathrm{~s}, 3 \mathrm{H}), 3.17(\mathrm{~s}, 3 \mathrm{H}), 3.01(\mathrm{t}, J=6.4 \mathrm{~Hz}$, $2 \mathrm{H}), 2.47(\mathrm{~s}, 3 \mathrm{H}) ;{ }^{13} \mathrm{C}$ NMR: $\delta$ 172.47, 156.95, 153.04, $150.28,148.19,145.48,139.84,138.45,133.68,132.14$, $131.27,129.59,129.50,128.03,127.94,126.38,124.68$, 122.63, 121.83, 108.37, 106.30, 102.57, 61.04, 57.46, 52.32, 26.63, 21.60; HRMS-ESI: $m / z$ calcd $426.16998 \mathrm{C}_{27} \mathrm{H}_{24} \mathrm{NO}_{4} \mathrm{Cl}$ $[\mathrm{M}-\mathrm{Cl}]^{+}$, found 462.16986 .

\section{8-(1-Naphthyl)protoberberine choride (6d)}

Yield: $72 \%$; Brown solid; mp $175-176^{\circ} \mathrm{C}$; ${ }^{1} \mathrm{H}$ NMR: $\delta 8.82$ (s, 1H), 8.15-8.32 (m, 3H), 7.95-8.07 (m, 1H), 7.64-8.00 (m, 5H), 7.45-7.55 (m, 1H), $7.03(\mathrm{~s}, 1 \mathrm{H}), 6.21(\mathrm{~s}, 2 \mathrm{H}), 4.11$ $-4.30(\mathrm{~m}, 2 \mathrm{H}), 4.10(\mathrm{~s}, 3 \mathrm{H}), 3.98$ (s, 3H), 2.90-3.05 $(\mathrm{m}, 2 \mathrm{H}) ;{ }^{13} \mathrm{C}$ NMR: $\delta$ 172.49, 156.87, 152.61, 150.73, $148.43,145.38,139.24,136.72,132.16,131.87,130.76$, $130.08,129.20,128.26,127.56,126.01,125.12,124.39$, $123.76,121.65,120.95,108.39,106.68,106.29,106.09$, 102.69, 60.99, 57.91, 52.56, 26.81; HRMS-ESI: $\mathrm{m} / z$ calcd $462.16998 \mathrm{C}_{30} \mathrm{H}_{24} \mathrm{NO}_{4} \mathrm{Cl}[\mathrm{M}-\mathrm{Cl}]^{+}$, found 462.16996 .

\section{8-(n-Decyl)protoberberine chloride (6e)}

Yield: $80 \%$; Brown solid; mp $185-186^{\circ} \mathrm{C} ;{ }^{1} \mathrm{H}$ NMR: $\delta$ $8.81(\mathrm{~s}, 1 \mathrm{H}), 8.20(\mathrm{~d}, J=9.2 \mathrm{~Hz}, 1 \mathrm{H}), 8.02(\mathrm{~d}, J=9.2 \mathrm{~Hz}$, $1 \mathrm{H}), 7.74(\mathrm{~s}, 1 \mathrm{H}), 7.12(\mathrm{~s}, 1 \mathrm{H}), 6.17(\mathrm{~s}, 2 \mathrm{H}), 4.80(\mathrm{t}, J=$ $6.4 \mathrm{~Hz}, 2 \mathrm{H}), 4.03(\mathrm{~s}, 3 \mathrm{H}), 4.05(\mathrm{~s}, 3 \mathrm{H}), 3.16(\mathrm{t}, J=6.4 \mathrm{~Hz}$, $2 \mathrm{H}), 1.78(\mathrm{t}, J=6.4 \mathrm{~Hz}, 2 \mathrm{H}), 1.27-1.58(\mathrm{~m}, 16 \mathrm{H}), 0.87(\mathrm{t}$, $J=6.4 \mathrm{~Hz}, 3 \mathrm{H}) ;{ }^{13} \mathrm{C}$ NMR: $\delta 172.49,161.58,152.99$, $150.09,148.11,146.08,138.29,133.10,131.28,125.66$, $125.20,121.92,120.71,108.19,106.26,102.48,62.05$, $57.52,50.10,32.77,31.78,29.83,29.46,29.22,29.19$, 28.40, 27.13, 22.58, 21.54, 14.45; HRMS-ESI: $m / z$ calcd 476.27954 $\mathrm{C}_{30} \mathrm{H}_{38} \mathrm{NO}_{4} \mathrm{Cl}[\mathrm{M}-\mathrm{Cl}]^{+}$, found 476.27971.

\section{8-(p-Methoxy)phenylpseudoprotoberberine choride (7a)}

Yield: $79 \%$; Bright orange solid; mp $197-198^{\circ} \mathrm{C} ;{ }^{1} \mathrm{H}$ NMR: $\delta$ $8.87(\mathrm{~s}, 1 \mathrm{H}), 7.79(\mathrm{~s}, 1 \mathrm{H}), 7.68(\mathrm{~s}, 1 \mathrm{H}), 7.66(\mathrm{~d}, J=8.4 \mathrm{~Hz}$, $2 \mathrm{H}), 7.33(\mathrm{~d}, J=8.4 \mathrm{~Hz}, 2 \mathrm{H}), 7.07(\mathrm{~s}, 1 \mathrm{H}), 6.70(\mathrm{~s}, 1 \mathrm{H}), 6.19$ $(\mathrm{s}, 2 \mathrm{H}), 4.34(\mathrm{t}, J=6.4 \mathrm{~Hz}, 2 \mathrm{H}), 4.09(\mathrm{~s}, 3 \mathrm{H}), 3.92(\mathrm{~s}, 3 \mathrm{H})$, $3.71(\mathrm{~s}, 3 \mathrm{H}), 3.04(\mathrm{t}, J=6.4 \mathrm{~Hz}, 2 \mathrm{H}) ;{ }^{13} \mathrm{C}$ NMR: $\delta 161.47$, $157.40,155.03,152.41,150.32,148.17,139.55,136.77$, $131.66,131.56,131.39,123.39,122.89,121.94,119.24$, $116.98,115.36,108.50,106.94,106.22,106.13,102.55$, 57.23, 56.27, 55.99, 52.12, 26.82; HRMS-ESI: $m / z$ calcd $442.16490 \mathrm{C}_{27} \mathrm{H}_{24} \mathrm{NO}_{5} \mathrm{Cl}[\mathrm{M}-\mathrm{Cl}]^{+}$, found 442.16488. 


\section{8-(m-Methoxy)phenylpseudoprotoberberine choride (7b)}

Yield: $77 \%$; Yellow solid; $\mathrm{mp} 224-225^{\circ} \mathrm{C}$; ${ }^{1} \mathrm{H}$ NMR: $\delta$ 8.90 (s, 1H), $7.80(\mathrm{~s}, 1 \mathrm{H}), 7.67-7.71$ (m, 2H), 7.30-7.35 $(\mathrm{m}, 2 \mathrm{H}), 7.26(\mathrm{~d}, J=6.4 \mathrm{~Hz}, 1 \mathrm{H}), 7.08(\mathrm{~s}, 1 \mathrm{H}), 6.66$ $(\mathrm{s}, 1 \mathrm{H}), 6.19(\mathrm{~s}, 2 \mathrm{H}), 4.33(\mathrm{t}, J=6.4 \mathrm{~Hz}, 2 \mathrm{H}), 4.09(\mathrm{~s}$, $3 \mathrm{H}), 3.85(\mathrm{~s}, 3 \mathrm{H}), 3.70(\mathrm{~s}, 3 \mathrm{H}), 3.06(\mathrm{t}, J=6.4 \mathrm{~Hz}, 2 \mathrm{H})$; ${ }^{13} \mathrm{C}$ NMR: $\delta$ 160.14, 157.53, 154.44, 152.48, 150.37, $148.20,139.48,136.92,132.26,131.74,131.34,122.93$, $121.79,121.62,119.33,117.31,115.15,108.54,106.72$, 106.20, 106.08, 102.57, 57.25, 56.27, 55.99, 52.27, 26.78; HRMS-ESI: $m / z$ calcd $442.16490 \mathrm{C}_{27} \mathrm{H}_{24} \mathrm{NO}_{5} \mathrm{Cl}[\mathrm{M}-\mathrm{Cl}]^{+}$, found 442.16475 .

\section{8-(p-Methyl)phenylpseudoprotoberberine choride (7c)}

Yield: 78\%; Yellow solid; mp $212-213{ }^{\circ} \mathrm{C} ;{ }^{1} \mathrm{H}$ NMR: $\delta 8.89$ (s, 1H), 7.80 (s, 1H), 7.69 (s, 1H), $7.61(\mathrm{~d}, J=8.4 \mathrm{~Hz}, 2 \mathrm{H})$, $7.58(\mathrm{~d}, J=8.4 \mathrm{~Hz}, 2 \mathrm{H}), 7.07(\mathrm{~s}, 1 \mathrm{H}), 6.65(\mathrm{~s}, 1 \mathrm{H}), 6.19$ (s, $2 \mathrm{H}), 4.31(\mathrm{t}, J=6.4 \mathrm{~Hz}, 2 \mathrm{H}), 4.09(\mathrm{~s}, 3 \mathrm{H}), 3.69(\mathrm{~s}, 3 \mathrm{H}), 3.05$ $(\mathrm{t}, J=6.4 \mathrm{~Hz}, 2 \mathrm{H}), 2.54(\mathrm{~s}, 3 \mathrm{H}) ;{ }^{13} \mathrm{C} \mathrm{NMR}: \delta 157.44$, $155.01,152.43,150.34,146.65,148.18,141.42,139.55$, $136.85,131.35,130.50,129.66,128.20,123.13,121.87$, $119.30,108.51,106.80,106.21,106.14,102.56,57.24$, 56.25, 52.20, 26.78, 21.64, 21.52; HRMS-ESI: $\mathrm{m} / z$ calcd $426.16998 \mathrm{C}_{27} \mathrm{H}_{24} \mathrm{NO}_{4} \mathrm{Cl}[\mathrm{M}-\mathrm{Cl}]^{+}$, found 426.16986 .

\section{8-(p-Chloro)phenylpseudoprotoberberine chloride (7d)}

Yield: $75 \%$; Bright orange solid; mp $183-184^{\circ} \mathrm{C}$; ${ }^{1} \mathrm{H}$ NMR: $\delta 8.91(\mathrm{~s}, 1 \mathrm{H}), 7.94(\mathrm{~d}, J=8.0 \mathrm{~Hz}, 2 \mathrm{H}), 7.87(\mathrm{~d}, J=8.0 \mathrm{~Hz}$, 2H), 7.75-7.80 (m, 1H) $7.69(\mathrm{~s}, 1 \mathrm{H}), 7.08(\mathrm{~s}, 1 \mathrm{H}), 6.60$ $(\mathrm{s}, 1 \mathrm{H}), 6.19(\mathrm{~s}, 2 \mathrm{H}), 4.30(\mathrm{t}, J=6.4 \mathrm{~Hz}, 2 \mathrm{H}), 4.09(\mathrm{~s}, 3 \mathrm{H})$, $3.72(\mathrm{~s}, 3 \mathrm{H}), 3.06(\mathrm{t}, J=6.4 \mathrm{~Hz}, 2 \mathrm{H}) ;{ }^{13} \mathrm{C}$ NMR: $\delta 157.55$, $153.59,152.59,150.41,148.22,139.64,136.97,134.87$, $132.64,131.83,130.97,130.20,129.89,126.50,123.05$, $121.76,119.55,108.56,106.50,106.22,105.06,102.60$, 57.28, 56.39, 52.38, 26.76; HRMS-ESI: $m / z$ calcd 446.11536 $\mathrm{C}_{26} \mathrm{H}_{21} \mathrm{NO}_{4} \mathrm{Cl}_{2}[\mathrm{M}-\mathrm{Cl}]^{+}$, found 446.11483.

\section{8-(m-Chloro)phenylpseudoprotoberberine chloride (7e)}

Yield: $76 \%$; Bright yellow solid; mp $179-180^{\circ} \mathrm{C}$; ${ }^{1} \mathrm{H}$ NMR: $\delta 8.93(\mathrm{~s}, 1 \mathrm{H}), 7.79-7.90(\mathrm{~m}, 5 \mathrm{H}), 7.71(\mathrm{~s}, 1 \mathrm{H})$, $7.08(\mathrm{~s}, 1 \mathrm{H}), 6.57(\mathrm{~s}, 1 \mathrm{H}), 6.20(\mathrm{~s}, 2 \mathrm{H}), 4.32(\mathrm{t}, J=6.4 \mathrm{~Hz}$, $2 \mathrm{H}), 4.09(\mathrm{~s}, 3 \mathrm{H}), 3.71(\mathrm{~s}, 3 \mathrm{H}), 3.07(\mathrm{t}, J=6.4 \mathrm{~Hz}, 2 \mathrm{H})$; ${ }^{13} \mathrm{C}$ NMR: $\delta$ 157.58, 152.94, 152.63, 150.44, 148.23, $139.58,137.05,134.69,132.96,131.98,131.74,131.35$, $129.59,128.64,123.00,121.69,119.63,108.57,106.40$, 106.23, 106.14, 102.61, 57.31, 56.36, 52.46, 26.76; HRMSESI: $m / z$ calcd $446.11536 \mathrm{C}_{26} \mathrm{H}_{21} \mathrm{NO}_{4} \mathrm{Cl}_{2}[\mathrm{M}-\mathrm{Cl}]^{+}$, found 446.11481.

\section{8-(1-Naphthyl)pseudoprotoberberine choride (7f)}

Yield: 72\%; Yellow-brown solid; $\mathrm{mp} \quad 198-200^{\circ} \mathrm{C}$ (decomp); ${ }^{1} \mathrm{H}$ NMR: $\delta 9.14(\mathrm{~s}, 1 \mathrm{H}), 8.38$ (d, $J=7.8 \mathrm{~Hz}$, $1 \mathrm{H}), 8.22(\mathrm{~d}, J=7.8 \mathrm{~Hz}, 1 \mathrm{H}), 7.84-7.92(\mathrm{~m}, 4 \mathrm{H}), 7.67$ $-7.71(\mathrm{~m}, 1 \mathrm{H}), 7.51-7.55(\mathrm{~m}, 1 \mathrm{H}), 7.40(\mathrm{~d}, J=8.2 \mathrm{~Hz}$,
$1 \mathrm{H}), 7.05(\mathrm{~s}, 1 \mathrm{H}), 6.34(\mathrm{~s}, 1 \mathrm{H}), 6.20(\mathrm{~s}, 2 \mathrm{H}), 4.20-4.32$ (m, 2H), 4.10 (s, 3H), $3.43(\mathrm{~s}, 3 \mathrm{H}), 2.93-3.05(\mathrm{~m}, 2 \mathrm{H})$; ${ }^{13}$ C NMR: $\delta$ 157.14, 152.66, 152.16, 149.89, 147.72, $139.79,136.83,133.09,131.62,130.90,130.17,129.01$, $128.92,128.36,127.50,127.35,125.86,124.42,123.19$, $121.54,119.55,108.07,105.90,105.80,105.65,102.12$, $56.85,55.65,51.55,26.40$; HRMS-ESI: $m / z$ calcd $462.17053 \mathrm{C}_{30} \mathrm{H}_{24} \mathrm{NO}_{4} \mathrm{Cl}[\mathrm{M}-\mathrm{Cl}]^{+}$, found 462.17072 (See Additional file 1).

\section{8-(n-Decyl)pseudoprotoberberine chloride (7g)}

Yield: 78\%; Yellow-brown solid; mp $173-174{ }^{\circ} \mathrm{C} ;{ }^{1} \mathrm{H}$ NMR: $\delta 8.67(\mathrm{~s}, 1 \mathrm{H}), 7.68(\mathrm{~s}, 2 \mathrm{H}), 7.11(\mathrm{~s}, 1 \mathrm{H}), 6.37(\mathrm{~s}, 1 \mathrm{H}), 6.17$ $(\mathrm{s}, 2 \mathrm{H}), 4.77(\mathrm{t}, J=6.4 \mathrm{~Hz}, 2 \mathrm{H}), 4.06(\mathrm{~s}, 6 \mathrm{H}), 3.67(\mathrm{t}, J=6.4$ $\mathrm{Hz}, 2 \mathrm{H},), 3.15(\mathrm{t}, J=6.4 \mathrm{~Hz}, 2 \mathrm{H}), 1.24-1.16(\mathrm{~m}, 16 \mathrm{H}), 0.85$ $(\mathrm{t}, J=4.0 \mathrm{~Hz}, 3 \mathrm{H}) ;{ }^{13} \mathrm{C}$ NMR: $\delta 158.04,157.19,152.67$, $150.12,148.07,139.33,136.11,131.31,122.12,122.02$, $118.54,108.31,106.60,106.21,106.15,102.49,57.11$, 56.94, 49.80, 31.77, 29.54, 29.45, 29.43, 29.26, 29.18 (2), 28.22, 27.17, 22.57, 14.44; HRMS-ESI: $m / z$ calcd 476.28008 $\mathrm{C}_{30} \mathrm{H}_{38} \mathrm{NO}_{4} \mathrm{Cl}[\mathrm{M}-\mathrm{Cl}]^{+}$, found 476.27990 (See Additional file 1).

\section{8-(n-Dodecyl)pseudoprotoberberine chloride (7h)}

Yield: $80 \%$; Bright yellow solid; mp $161-163^{\circ} \mathrm{C}$ (decomp); ${ }^{1} \mathrm{H}$ NMR: $\delta 8.65$ (s, 1H), $7.66(\mathrm{~s}, 1 \mathrm{H}), 7.59(\mathrm{~s}, 1 \mathrm{H}), 7.09$ $(\mathrm{s}, 1 \mathrm{H}), 6.36(\mathrm{~s}, 1 \mathrm{H}), 6.16(\mathrm{~s}, 2 \mathrm{H}), 4.75(\mathrm{t}, J=6.4 \mathrm{~Hz}, 2 \mathrm{H})$, $4.05(\mathrm{~s}, 6 \mathrm{H}), 3.65(\mathrm{t}, J=6.4 \mathrm{~Hz}, 2 \mathrm{H}),, 3.14(\mathrm{t}, J=6.4 \mathrm{~Hz}$, $2 \mathrm{H}), 1.20-1.74(\mathrm{~m}, 20 \mathrm{H}), 0.84(\mathrm{t}, J=4.0 \mathrm{~Hz}, 3 \mathrm{H}) ;{ }^{13} \mathrm{C}$ NMR: $\delta$ 158.12, 157.18, 152.67, 150.12, 148.07, 139.30, $136.09,131.28,122.09,121.99,118.50,108.30,106.57$, $106.17,106.12,102.50,57.10,56.93,49.79,31.78,29.53$, $29.49,29.42,29.32,29.25,29.17,29.14,28.84,28.21$, 27.16, 22.58, 14.44; HRMS-ESI: $\mathrm{m} / z$ calcd 504.31084 $\mathrm{C}_{32} \mathrm{H}_{42} \mathrm{NO}_{4} \mathrm{Cl}[\mathrm{M}-\mathrm{Cl}]^{+}$, found 504.31047.

\section{8-(p-Methoxy)phenylprotopalmatine choride (8a)}

Yield: $81 \%$; Brown solid; mp $168-169^{\circ} \mathrm{C}$; ${ }^{1} \mathrm{H}$ NMR: $\delta$ $9.13(\mathrm{~s}, 1 \mathrm{H}), 8.24(\mathrm{~d}, J=9.2 \mathrm{~Hz}, 1 \mathrm{H}), 8.14(\mathrm{~d}, J=9.2 \mathrm{~Hz}$, $1 \mathrm{H}), 7.67$ (s, 1H), $7.58(\mathrm{~d}, J=8.2 \mathrm{~Hz}, 2 \mathrm{H}), 7.23(\mathrm{~d}, J=8.2$ $\mathrm{Hz}, 2 \mathrm{H}), 7.04(\mathrm{~s}, 1 \mathrm{H}), 4.29(\mathrm{t}, J=6.4 \mathrm{~Hz}, 2 \mathrm{H}), 4.00(\mathrm{~s}$, $3 \mathrm{H}), 3.96(\mathrm{~s}, 3 \mathrm{H}), 3.86(\mathrm{~s}, 3 \mathrm{H}), 3.82(\mathrm{~s}, 3 \mathrm{H}), 3.24(\mathrm{~s}, 3 \mathrm{H})$, $3.05(\mathrm{t}, J=6.4 \mathrm{~Hz}, 2 \mathrm{H}) ;{ }^{13} \mathrm{C}$ NMR: $\delta$ 172.49, 160.74, $156.93,152.77,151.98,149.36,145.94,139.98,138.68$, $133.77,131.53,129.72,126.78,124.63,122.78,121.49$, $120.32,113.38,114.51,111.29,109.69,61.32,57.96$, 56.81, 56.42, 55.87, 55.92, 26.37; HRMS-ESI: $m / z$ calcd $458.19620 \mathrm{C}_{28} \mathrm{H}_{28} \mathrm{NO}_{5} \mathrm{Cl}[\mathrm{M}-\mathrm{Cl}]^{+}$, found 458.19613 .

\section{8-(m-Methoxy)phenylprotopalmatine choride (8b)}

Yield: 78\%; Red-brown solid; mp $170-171^{\circ} \mathrm{C} ;{ }^{1} \mathrm{H}$ NMR: $\delta$ $9.15(\mathrm{~s}, 1 \mathrm{H}), 8.25(\mathrm{~d}, J=9.2 \mathrm{~Hz}, 1 \mathrm{H}), 8.14(\mathrm{~d}, J=9.2 \mathrm{~Hz}$, $1 \mathrm{H}), 7.76(\mathrm{~s}, 1 \mathrm{H}), 7.56-7.60(\mathrm{~m}, 1 \mathrm{H}), 7.20-7.26(\mathrm{~m}, 3 \mathrm{H})$, $7.05(\mathrm{~s}, 1 \mathrm{H}), 4.29(\mathrm{t}, J=6.4 \mathrm{~Hz}, 2 \mathrm{H}), 4.00(\mathrm{~s}, 3 \mathrm{H}), 3.96(\mathrm{~s}$, 
$3 \mathrm{H}), 3.86(\mathrm{~s}, 3 \mathrm{H}), 3.82(\mathrm{~s}, 3 \mathrm{H}), 3.24(\mathrm{~s}, 3 \mathrm{H}), 3.05(\mathrm{t}, J=$ 6.4 Hz, 2H); ${ }^{13} \mathrm{C}$ NMR: $\delta$ 165.70, 159.62, 156.25, 152.87, $152.04,149.24,145.37,138.59,136.24,133.82,130.42$, $129.30,126.42,124.54,122.36,121.52,120.10,115.78$, $113.73,111.21,109.66,61.13,57.42,56.73,56.34,55.87$, $52.49,26.35$; HRMS-ESI: $m / z$ calcd $458.19619 \mathrm{C}_{28} \mathrm{H}_{28} \mathrm{NO}_{5} \mathrm{Cl}$ $[\mathrm{M}-\mathrm{Cl}]^{+}$, found 458.19615 .

\section{8-(p-Methyl)phenylprotopalmatine choride (8c)}

Yield: 74\%; Yellow-brown solid; mp $164-165^{\circ} \mathrm{C}$; ${ }^{1} \mathrm{H}$ NMR: $\delta 9.15(\mathrm{~s}, 1 \mathrm{H}), 8.24(\mathrm{~d}, J=9.2 \mathrm{~Hz}, 1 \mathrm{H}), 8.15(\mathrm{~d}, J=$ $9.2 \mathrm{~Hz}, 1 \mathrm{H}), 7.76(\mathrm{~s}, 1 \mathrm{H}), 7.46-7.52(\mathrm{~m}, 4 \mathrm{H}), 7.04(\mathrm{~s}$, $1 \mathrm{H}), 4.25(\mathrm{t}, J=6.4 \mathrm{~Hz}, 2 \mathrm{H}), 4.00(\mathrm{~s}, 3 \mathrm{H}), 3.96(\mathrm{~s}, 3 \mathrm{H})$, $3.86(\mathrm{~s}, 3 \mathrm{H}), 3.18(\mathrm{~s}, 3 \mathrm{H}), 3.03(\mathrm{t}, J=6.4 \mathrm{~Hz}, 2 \mathrm{H}), 2.48(\mathrm{~s}$, $3 \mathrm{H}) ;{ }^{13} \mathrm{C}$ NMR: $\delta 172.49,156.94,152.89,152.01,149.22$, $145.44,139.80,138.66,133.78,132.22,129.51,129.29$, $128.00,127.93,126.38,124.57,122.56,121.47,120.26$, 111.19, 109.67, 61.02, 57.43, 56.74, 56.34, 52.45, 26.32, 21.59; HRMS-ESI: $m / z$ calcd $442.20128 \mathrm{C}_{28} \mathrm{H}_{28} \mathrm{NO}_{4} \mathrm{Cl}$ $[\mathrm{M}-\mathrm{Cl}]^{+}$, found 442.20128.

\section{8-(1-Naphthyl)protopalmatine choride (8d)}

Yield: 78\%; Yellow-brown solid; mp $177-178^{\circ} \mathrm{C}$; ${ }^{1} \mathrm{H}$ NMR: $\delta 9.34$ (s, 1H), 8.20-8.25 (m, 3H), 8.18 (d, $J=8.4$ $\mathrm{Hz}, 1 \mathrm{H}), 7.84(\mathrm{~s}, 1 \mathrm{H}), 7.76-7.80(\mathrm{~m}, 2 \mathrm{H}), 7.63-7.67(\mathrm{~m}$, $1 \mathrm{H}), 7.47-7.49(\mathrm{~m}, 2 \mathrm{H}), 7.02(\mathrm{~s}, 1 \mathrm{H}), 4.13-4.35(\mathrm{~m}, 2 \mathrm{H})$, $3.99(\mathrm{~s}, 3 \mathrm{H}), 3.94(\mathrm{~s}, 3 \mathrm{H}), 3.85(\mathrm{~s}, 3 \mathrm{H}), 2.93-3.05(\mathrm{~m}$, 2H), 2.77 (s, 3H); ${ }^{13} \mathrm{C}$ NMR: $\delta 172.49,155.06,152.70$, $152.07,149.25,144.94,139.44,134.03,133.13,132.42$, $130.87,130.58,129.29,128.21,127.47,126.59,126.44$, $126.00,125.06,124.74,123.15,122.16,120.41,111.19$, $109.68,60.78,57.35,56.78,56.35,52.40,26.59$; HRMSESI: $m / z$ calcd $478.20128 \mathrm{C}_{31} \mathrm{H}_{28} \mathrm{NO}_{4} \mathrm{Cl}[\mathrm{M}-\mathrm{Cl}]^{+}$, found 478.20113 .

\section{8-(n-Decyl)protopalmatine chloride (8e)}

Yield: $75 \%$; Brown solid; mp $172-173^{\circ} \mathrm{C}$; ${ }^{1} \mathrm{H}$ NMR: $\delta$ $8.90(\mathrm{~s}, 1 \mathrm{H}), 8.20(\mathrm{~d}, J=9.2 \mathrm{~Hz}, 1 \mathrm{H}), 8.02(\mathrm{~d}, J=9.2 \mathrm{~Hz}$, $1 \mathrm{H}), 7.65(\mathrm{~s}, 1 \mathrm{H}), 7.13(\mathrm{~s}, 1 \mathrm{H}), 4.83(\mathrm{t}, J=6.4 \mathrm{~Hz}, 2 \mathrm{H})$, $4.07(\mathrm{~s}, 3 \mathrm{H}), 4.05(\mathrm{~s}, 3 \mathrm{H}), 3.93(\mathrm{~s}, 3 \mathrm{H}), 3.88(\mathrm{~s}, 3 \mathrm{H}), 3.18$ $(\mathrm{t}, J=6.4 \mathrm{~Hz}, 2 \mathrm{H}), 1.79(\mathrm{t}, J=6.4 \mathrm{~Hz}, 2 \mathrm{H}), 1.27-1.60(\mathrm{~m}$, $16 \mathrm{H}), 0.87(\mathrm{t}, J=6.4 \mathrm{~Hz}, 3 \mathrm{H}) ;{ }^{13} \mathrm{C}$ NMR: $\delta 176.25$, $161.55,152.84,151.86,149.18,146.05,138.49,133.20$, $129.31,125.67,125.08,121.62,120.35,111.06,109.62$, $62.04,57.50,56.68,56.36,50.23,32.77,31.78,29.84$, 29.48, 29.46, 29.23, 29.19, 28.43, 26.84, 22.58, 14.45; HRMS-ESI: $m / z$ calcd $492.31084 \mathrm{C}_{31} \mathrm{H}_{42} \mathrm{NO}_{4} \mathrm{Cl}[\mathrm{M}-\mathrm{Cl}]^{+}$, found 492.31064 .

\section{Biological activity assay}

18 newly synthesized analogues were evaluated for their activity against the multiplication of wild-type $M$. tuberculosis strain $\mathrm{H}_{37} \mathrm{Rv}$ and MRD strains by the microplate alamar blue assay (MABA) at various concentrations of
128.0, 64.0, 32.0, 16.0, 8.0, 4.0, 2.0, 1.0, 0.5, 0.25, 0.125, and $0.0625 \mu \mathrm{g} / \mathrm{ml}$. RIF and INH were used as positive controls. Subsequent two-fold dilutions were performed in $100 \mu \mathrm{L}$ of $7 \mathrm{H} 9$ media in the 48 -well microplates. Then $100 \mu \mathrm{L}$ of bacterial suspension was added to result in a final bacterial titer of $1 \times 10^{6} \mathrm{CFU} / \mathrm{mL}$. Plates were incubated at $37^{\circ} \mathrm{C}$. At optimal time, alamar blue solution was added to the entire plate. Results were recorded at $24 \mathrm{~h}$ post-reagent addition. Visual MIC value was defined as the lowest concentration of drug that prevented a color change.

\section{Cytotoxicity activity assay}

African green monkey kidney (Vero) cells $\left(6 \times 10^{3}\right.$ cells/ well) and human lung fibroblast (MRC-5) cells $\left(1.2 \times 10^{4}\right.$ cells/well) were plated into a 96-well plates and incubated $37^{\circ} \mathrm{C}$ in $5 \% \mathrm{CO}_{2}$. Sixteen hours later the cell cultures were treated with various concentrations of compounds $\mathbf{7 f}$ and $7 \mathrm{~g}$. Cytotoxicity was evaluated with the tetrazolium 3(4,5-dimethylthiazol-2-yl)-2,5-diphenyltetrazolium bromide (MTT) assay at $48 \mathrm{~h}$. The $50 \%$ cytotoxic concentration $\left(\mathrm{CC}_{50}\right)$ was calculated with Reed \& Muench methods. Each experiment was repeated three times.

\section{Conclusion}

In conclusion, 18 new 8-substituted BBR derivatives were synthesized and evaluated for their antimycobacterial activities against $M$. tuberculosis $\mathrm{H}_{37} \mathrm{Rv}$. SAR analysis revealed that (i) introduction of a $n$-decyl at the 8-position might significantly enhance the activity; (ii) 10,11dimethoxy on the ring $\mathrm{D}$ might be beneficial for the antimycobacterial activity. Among the test compounds, compound $7 \mathbf{g}$ exhibited the strongest activity against both drug-susceptible strains and MDR isolates of M. tuberculosis, suggesting a novel mechanism of action. It has been selected as an ideal compound lead against TB for further SAR investigation. We consider 8 - $n$-decylberberines to be a novel family of anti-tubercular agents with an advantage of inhibiting MDR strains of M. tuberculosis.

\section{Additional file}

Additional file 1: Supporting information. Selected copies of spectrum (1H-NMR, 13C-NMR and HRMS) for the two representative compounds.

\section{Competing interests}

The authors declare that they have no competing interests.

\section{Authors' contributions}

The current study is an outcome of the constructive discussion with DQS and YXW, who offered necessary guidance to YHL and HGF to carry out their synthesis and characterization experiments. FS and LMG performed the antimycobacterial activities against M. tuberculosis $\mathrm{H}_{37} \mathrm{Rv}$ tests, ST and CWB carried out the ${ }^{1} \mathrm{H}$ NMR and ${ }^{13} \mathrm{C}$ NMR spectral analyses and HRMS analysis, and YHL did the cytotoxicity experiment. All authors read and approved the final manuscript. 


\section{Acknowledgment}

This work was supported by the National Natural Science Fundation for Young Scientists (81102312) and the "National Mega-project for Innovative Drugs" (2012ZX09301002-001-017).

\section{Author details}

${ }^{1}$ Institute of Medicinal Biotechnology, Chinese Academy of Medical Sciences and Peking Union Medical College, Beijing 100050, China. ${ }^{2}$ College of

Chemical Engineering, Qingdao University of Science and Technology, Qingdao 266042, China.

Received: 1 April 2013 Accepted: 28 June 2013

Published: 10 July 2013

\section{References}

1. Butler D: New fronts in an old war. Nature 2000, 406:670-672.

2. Dye C, Williams BG: The population dynamics and control of tuberculosis. Science 2010, 328:856-861.

3. Koul A, Arnoult E, Lounis N, Guillemont J, Andries K: The challenge of new drug discovery for tuberculosis. Nature 2011, 469:483-490.

4. Jiao WW, Mokrousov I, Sun GZ, Li M, Liu JW, Narvskaya O, et al: Molecular characteristics of rifampin and isoniazid resistant mycobacterium tuberculosis strains from beijing. China. Chin Med J (Engl) 2007, 120:814-819.

5. Zhao Y, Xu S, Wang L, Chin D, Wang S, Jiang G, et al: National survey of drug-resistant tuberculosis in china. N Engl I Med 2012, 366:2161-2170.

6. Verma SC, Dhungana GP, Joshi HS, Kunwar HB, Pokhrel AK: Prevalence of pulmonary tuberculosis among HIV infected persons in pokhara, nepal. J Nepal Health Res Counc 2012, 10:32-36.

7. Cain KP, Varma JK: Finding tuberculosis at the first encounter with HIV care: don't miss the opportunity to save a life. Int J Tuberc Lung Dis 2012 16:1138.

8. He JL, Xie JP: Advances in mycobacterium siderophore-based drug discovery. Acta Pharmaceutica Sinica B 2011, 1:8-13.

9. Tomioka H: Current status of some antituberculosis drugs and the development of new antituberculous agents with special reference to their in vitro and in vivo antimicrobial activities. Curr Pharm Des 2006, 12:4047-4070

10. Spigelman MK: New tuberculosis therapeutics: a growing pipeline. $J$ Infect Dis 2007, 196(Suppl 1):S28-34.

11. FDA: FDA news release; 2012. http://www.fda.gov/NewsEvents/Newsroom/ PressAnnouncements/ucm333695.htm.

12. Liu YX, Xiao CL, Wang YX, Li YH, Yang YH, Li YB, et al: Synthesis, structureactivity relationship and in vitro anti-mycobacterial evaluation of 13-n-octylberberine derivatives. Eur J Med Chem 2012, 52:151-158.

13. Yang P, Song DQ, Li YH, Kong WJ, Wang YX, Gao LM, et al: Synthesis and structure-activity relationships of berberine analogues as a novel class of low-density-lipoprotein receptor up-regulators. Bioorg Med Chem Lett 2008, 18:4675-4677.

14. Wang YX, Wang YP, Zhang H, Kong WJ, Li YH, Liu F, et al: Synthesis and biological evaluation of berberine analogues as novel up-regulators for both low-density-lipoprotein receptor and insulin receptor. Bioorg Med Chem Lett 2009, 19:6004-6008.

15. Yang $Y, Y e X L$, Li XG, Zhen J, Zhang B, Yuan L: Synthesis and antimicrobial activity of 8-alkylberberine derivatives with a long aliphatic chain Planta Med 2007, 73:602-604.

16. Iwasa K, Lee DU, Kang SI, Wiegrebe W: Antimicrobial activity of 8-alkyl- and 8-phenyl-substituted berberines and their 12-bromo derivatives. J Nat Prod 1998, 61:1150-1153.

doi:10.1186/1752-153X-7-117

Cite this article as: $L i$ et al: Synthesis and structure-activity relationship of 8-substituted protoberberine derivatives as a novel class of antitubercular agents. Chemistry Central Journal 2013 7:117.

\section{Publish with ChemistryCentral and every scientist can read your work free of charge \\ "Open access provides opportunities to our colleagues in other parts of the globe, by allowing anyone to view the content free of charge." W. Jeffery Hurst, The Hershey Company. \\ - available free of charge to the entire scientific community \\ - peer reviewed and published immediately upon acceptance \\ - cited in PubMed and archived on PubMed Central \\ - yours - you keep the copyright \\ Submit your manuscript here: \\ http://www.chemistrycentral.com/manuscript/<smiles>c1ccccc1</smiles> \\ Chemistry Central}

